\title{
Original
}

\section{Comparing the Progression of Hip Joint Destruction in Patients with Rheumatoid Arthritis before and after Intervention with Methotrexate and Biologics DMARDs}

\author{
Yoichi Toyoshima ${ }^{1)}$, Fumio SuKeZaki ${ }^{1)}$, Koei $\mathrm{OH}^{2)}$, \\ Masanori NAKAMURA ${ }^{2)}$, Hideyo MiYaOKA ${ }^{1)}$ and Katsunori INAGAKI ${ }^{1)}$
}

\begin{abstract}
We investigated hip joint destruction in patients with rheumatoid arthritis before and after the availability of methotrexate and biological disease-modifying anti-rheumatic drugs (DMARDs) in Japan. A total of 35 patients with 45 diseased joints underwent total hip arthroplasty from 1995 to 1999 (Group A: 21 hips in 15 patients) and from 2006 to 2010 (Group B: 24 hips in 20 patients). We monitored the oral administration of therapeutic drugs and prednisolone, measured preoperative C-reactive protein (CRP) levels, estimated the Larsen grade and bone defects of the acetabulum floor using simple X-ray images, and calculated the bone defect rate of the femoral head. The intake of preoperative oral prednisolone was significantly less in Group B than in Group A $(P<0.05)$, as were preoperative CRP levels $(P<0.05)$. There was no difference in Larsen grade between the groups. Type III (combined type) bone defects of a large size in the acetabulum base were observed in the hip joints of 12 hips in Group A (57.1\%) and 10 hips in Group B $(41.7 \%)$, and the mean rate of bone defects in the femoral head was $26.7 \%$ in Group A and $33.7 \%$ in Group B. These findings indicated that the recent introduction of effective medical treatment has decreased the number of patients with large bone defects in the acetabulum. Furthermore, the number of patients subjected to total hip arthroplasty for rheumatoid arthritis accompanied by severe hip joint destruction has decreased. However, the number of patients who underwent total hip arthroplasty was slightly higher in this study, indicating that even if the disease activity of rheumatoid arthritis is suppressed, joint destruction may continue in the hip joint of patients with previously recognized mild joint destruction.
\end{abstract}

Key words: rheumatoid arthritis, hip joint destruction, methotrexate, biologics, total hip arthroplasty

\section{Introduction}

Effective medical treatment for rheumatoid arthritis can prevent the progression of joint destruction. However, to our knowledge, preventing destruction of the weight-bearing hip has

\footnotetext{
1) Department of Orthopaedic Surgery, Showa University School of Medicine, 1-5-8 Hatanodai, Shinagawa-ku Tokyo, 142-8666, Japan.

2) Department of Orthopaedic Surgery, Showa University Northern Yokohama Hospital.
} 
not been reported. We investigated patients who underwent total hip arthroplasty to evaluate whether therapeutic interventions such as methotrexate or biological disease-modifying antirheumatic drugs (DMARDs) could inhibit destruction of the hip joint.

\section{Patients and methods}

Patients with rheumatoid arthritis requiring total hip arthroplasty for progressive hip joint destruction were recruited for this study (35 patients, 45 affected hip joints). Group A (15 patients, 21 affected joints) underwent surgery between January 1995 and December 1999, whereas Group B (20 patients, 24 affected joints) underwent surgery between January 2006 and December 2010. There were no differences in age or disease duration between Groups A and B (Table 1).

We evaluated the preoperative medical treatments and volumes of oral prednisolone intake (prednisolone conversion) administered to the patients in this study. In addition, we assessed disease activity on the basis of preoperative serum C-reactive protein (CRP) levels, evaluated the degree of joint destruction based on Larsen grade and simple X-ray images, examined the degree of bone destruction of the acetabulum floor according to the American Academy of Orthopaedic Surgeons (AAOS) classification, and measured the percentage of defective bone in the femoral head using the Steinberg method ${ }^{1-3)}$.

Our first choice of procedure for rheumatoid hips was a cementless total-hip arthroplasty. We recorded the use of bone grafts (autogenous and allogeneic bone grafts) and cup supporters for reconstructing the acetabular bone defects.

\section{Statistical analysis}

Differences between groups were analyzed using the two-tailed unpaired Student's t-test. Nonparametric data were evaluated with the Mann-Whitney $U$ test. Significance was set at $P<0.05$.

\section{Results}

Group A patients used conventional DMARDs, including bucillamine (BUC), as well

Table 1. Patients

\begin{tabular}{ccc}
\hline & Group A & Group B \\
& Jan, 1995-Dec, 1999 & Jan, 2006-Dec, 2010 \\
\hline Patient, Joint & 15 patients, 21 hips & 20 patients, 24 hips \\
Gender (Male: Female) & 2: 13 & $5: 15$ \\
Age (y) & Mean 59.0 $(42-76)$ & Mean $63.8(34-81)$ \\
Disease duration (y) & Mean $13.6(2-38)$ & Mean $14.8(3-38)$ \\
Steinblocker Stage & III : 10, IV : 5 & III : 12, IV : 8 \\
Steinblocker Class & $2: 1,3: 8,4: 6$ & $2: 4,3: 12,4: 4$ \\
\hline
\end{tabular}




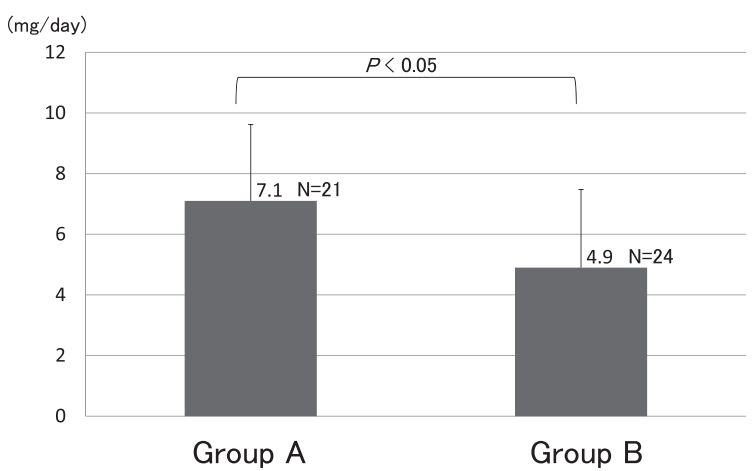

Fig. 1. Dose of oral prednisolone (preoperative) Preoperative oral administration of prednisolone differed significantly between the groups: mean 7.1 $+-2.7 \mathrm{mg} /$ day in Group A and $4.9+-2.6 \mathrm{mg} /$ day in Group B $(P<0.05)$.

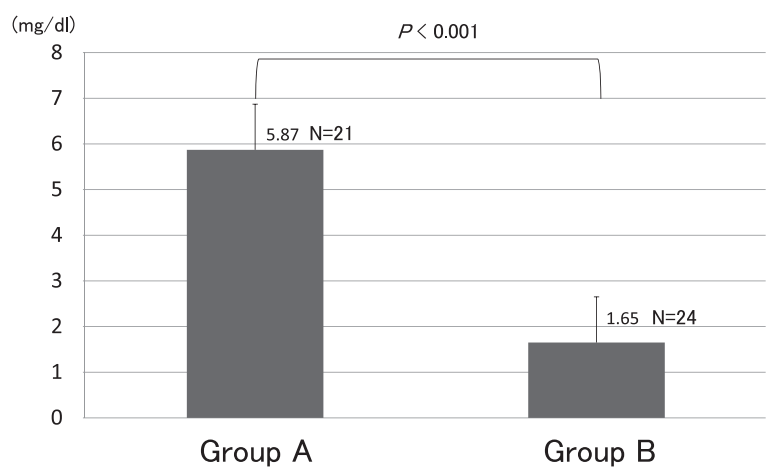

Fig. 2. Serum CRP level (preoperative) Preoperative CRP (mg/dL) levels were significantly higher in Group A $(5.87+-3.9 \mathrm{mg} / \mathrm{dL})$ than in Group B $(1.65+-2.09 \mathrm{mg} / \mathrm{dL} ; P<0.001)$.

Table 2. Bone defects in the acetabular floor

\begin{tabular}{|c|c|c|c|c|c|}
\hline & Grou & $\mathrm{p} A$ & $(\mathrm{~N}=21)$ & Group $\mathrm{E}$ & $(\mathrm{N}=24)$ \\
\hline \multirow[t]{3}{*}{ Larsen grade } & Grade & 3 & $2(9.5 \%)$ & 3 & $5(20.8 \%)$ \\
\hline & & 4 & $16(76.2 \%)$ & 4 & $16(66.7 \%)$ \\
\hline & & 5 & $3(14.3 \%)$ & 5 & $3(12.52 \%)$ \\
\hline \multirow[t]{4}{*}{ AAOS classification } & Type & I & $6(28.5 \%)$ & I & $9(37.5 \%)$ \\
\hline & & II & $3(14.3 \%)$ & II & $5(20.8 \%)$ \\
\hline & & III & $12(57.1 \%)$ & III & $10(41.7 \%)$ \\
\hline & IV, & $\mathrm{V}$ & 0 & IV, $\mathrm{V}$ & 0 \\
\hline
\end{tabular}

as various other drugs (BUC: 5 patients, MTX: 3 patients, salazosulfapyridine (SSZ): 2 patients, actarit: 3 patients, intramuscular or oral gold (GOLD): 3 patients, D-penicillamine: 2 patients). MTX was administered for less than 6 months in this group. In Group B, MTX was administered to 15 patients $(62.5 \%)$, whereas biologics were administered to 5 patients (infliximab: 3 patients, etanercept and tocilizumab: 1 patient). Other DMARDs used in Group B included BUC: 4 patients, SSZ: 2 patients, leflunomide: 2 patients, and oral GOLD: 1 patient.

Preoperative oral administration of prednisolone differed significantly between the groups, with a mean of $7.1+-2.7 \mathrm{mg} /$ day in Group A and $4.9+-2.6 \mathrm{mg} / \mathrm{day}$ in Group B (Fig. 1). Preoperative CRP $(\mathrm{mg} / \mathrm{dL})$ levels were also significantly higher in Group A $(5.87+-3.9 \mathrm{mg} / \mathrm{dL})$ than in Group B (1.65 +-2.09 mg/dL) (Fig. 2).

Larsen grades were similar in Groups A and B. When we evaluated bone defects in the acetabular floor using the AAOS Classification, type III defects were observed in 12 patients of Group A (57.1\%) and in 10 patients of Group B (41.7\%) (Table 2). Protrusion acetabula was observed in 8 Group A patients (38.1\%) and in 7 Group B patients (39.2\%). According to the Steinberg method, the bone defect rate in the femoral head averaged $26.7 \%$ in Group A 
(5-70\%) and 33.7\% in Group B (10-90\%).

Bone cement was used only in 2 patients, both in Group A. In both Groups, 12 patients required impaction bone grafts in the cementless cup, comprising chips obtained by smashing the excised femoral head. No allogeneic bone graft and cup supporter was used in this study.

\section{Discussion}

Decreased numbers of total joint arthroplasty procedures were reported recently due to advances in the medical treatment of rheumatoid arthritis. Furthermore, a new diagnostic classification and therapeutic algorithm has been suggested ${ }^{4-12)}$. We evaluated the degree of destruction in the hip joint among patients who underwent total hip arthroplasty before and after the use of methotrexate and biologics DMARDs was introduced in Japan.

In our study, we witnessed an increased number of patients who underwent total hip arthroplasty after the introduction of methotrexate and biologics DMARDs. This may be explained by improvements in available treatments over the previous years, leading to better prognosis and increased survival of patients with rheumatoid arthritis ${ }^{13)}$. The findings of this study also suggested that aggressive medical treatment like biologics DMARDs may limit the control of joint destruction in patients with weight-bearing joints in which changes have developed $^{14)}$. Although disease activity was suppressed by the biologics treatment, radiographic progression in weight-bearing joints occurred.

The treatment group in this study showed no change in Larsen grade with treatment, reflecting that hip joint destruction in our target population had progressed similarly among patients. Before the introduction of effective medical treatment, 12 hips (57.1\%) were classified as type III (AAOS classification), which reflects a large defect of the acetabulum floor in the hip joint. This incidence decreased to 10 hips (41.7\%) after the introduction of effective drug interventions, suggesting that medical treatment effectively suppresses bone destruction. Our investigation did not show any differences in the rate of bone defects in the femoral head between the groups. Disease activity in rheumatoid arthritis may therefore not be related to the degree of femoral head destruction ${ }^{15)}$.

No patients required allogeneic bone grafts or cup support, indicating that complete acetabulum floor reconstruction was achieved using autogenous bone grafts and large-diameter cups in all cases. This result could reflect the suppression of disease activity and bone destruction by the medical treatment as well as improved global patient assessment. Moreover, such improvements might have enhanced the desire for increased physical activity during daily life. Therefore, total hip arthroplasty might have been performed before the final stage of joint destruction.

In conclusion, bone defects in the acetabulum floor of the hip joint have been successfully ameliorated in recent years by total hip arthroplasty in patients with rheumatoid arthritis, and thus, the prevalence of such surgery has increased despite the introduction of effective medical treatment. These findings indicated that the destruction of weight-bearing joints continues even with pharmaceutical intervention. 


\section{Conflict of interest}

The authors declare no conflict of interest.

\section{References}

1) Larsen A, Dale K, Eek M. Radiographic evaluation of rheumatoid arthritis and related conditions by standard reference films. Acta Radiol Diagn (Stockh). 1977;18:481-491.

2) D'Antonio JA, Capello WN, Borden LS, et al. Classification and management of acetabular abnormalities in total hip arthroplasty. Clin Orthop Relat Res. 1989;243:126-137.

3) Steinberg DR, Steinberg ME, Garino JP, et al. Determining lesion size in osteonecrosis of the femoral head. $J$ Bone Joint Surg Am. 2006;88 Suppl 3:27-34.

4) Aletaha D, Neogi T, Silman AJ, et al. 2010 rheumatoid arthritis classification criteria: an American College of Rheumatology/European League Against Rheumatism collaborative initiative. Ann Rheum Dis. 2010;69:1580-1588.

5) Smolen JS, Landewe R, Breedveld FC, et al. EULAR recommendations for the management of rheumatoid arthritis with synthetic and biological disease-modifying antirheumatic drugs. Ann Rheum Dis. 2010;69:964-975.

6) Momohara S, Inoue $\mathrm{E}$, Ikari $\mathrm{K}$, et al. Decrease in orthopaedic operations, including total joint replacements, in patients with rheumatoid arthritis between 2001 and 2007: data from Japanese outpatients in a single institutebased large observational cohort (IORRA). Ann Rheum Dis. 2010;69:312-313.

7) Hekmat K, Jacobsson L, Nilsson JA, et al. Decrease in the incidence of total hip arthroplasties in patients with rheumatoid arthritis--results from a well defined population in south Sweden. Arthritis Res Ther. 2011;13:R67.

8) Sokka T, Kautiainen H, Hannonen P. Stable occurrence of knee and hip total joint replacement in Central Finland between 1986 and 2003: an indication of improved long-term outcomes of rheumatoid arthritis. Ann Rheum Dis. 2007;66:341-344.

9) da Silva E, Doran MF, Crowson CS, et al. Declining use of orthopedic surgery in patients with rheumatoid arthritis? Results of a long-term, population-based assessment. Arthritis Rheum. 2003;49:216-220.

10) Weiss RJ, Stark A, Wick MC, et al. Orthopaedic surgery of the lower limbs in 49802 rheumatoid arthritis patients: results from the Swedish National Inpatient Registry during 1987 to 2001. Ann Rheum Dis. 2006;65:335-341.

11) Louie GH, Ward MM. Changes in the rates of joint surgery among patients with rheumatoid arthritis in California, 1983-2007. Ann Rheum Dis. 2010;69:868-871.

12) Weiss RJ, Ehlin A, Montgomery SM, et al. Decrease of RA-related orthopaedic surgery of the upper limbs between 1998 and 2004: data from 54,579 Swedish RA inpatients. Rheumatology (Oxford). 2008;47:491-494.

13) Nakajima A, Inoue E, Tanaka E, et al. Mortality and cause of death in Japanese patients with rheumatoid arthritis based on a large observational cohort, IORRA. Scand J Rheumatol. 2010;39:360-367.

14) Seki E, Matsushita I, Sugiyama E, et al. Radiographic progression in weight-bearing joints of patients with rheumatoid arthritis after TNF-blocking therapies. Clin Rheumatol. 2009;28:453-460.

15) Bossingham DH, Schorn D, Morgan GW, et al. Progression of hip disease in rheumatoid arthritis. Rheumatol Rehabil. 1978;17:170-178.

[Received January 6, 2014: Accepted January 28, 2014] 